\title{
III.
}

\section{Das plexiforme Neurom (cylindrische Fibrom der Nervensclieiden).}

\author{
Von Dr. Richard Marchand, \\ Assistenten am physiologischen Institute der Universitaat IJalle a. S.
}

(Hierzu Taf. I.)

Die Kenntniss von den plexiformen Neuromen rührt erst aus der neueren Zeit her. Besonders in der jüngsten Vergangenheit wurden wiederholentlich Fälle davon beobachtet. Doch hat man sich bisher noch kein völlig klares Urtheil über die wesentlichen Eigenschaften und die Bedeutung dieser Geschwulstform bilden können. Auf den folgenden Seiten sollen nun zwei weitere Fälle beschrieben und soll demnächst der Versuch gemacht werden, das Wesentlicbe, was bisher uber Symptome, Bau und Entwickelung der Geschwulst in einzelnen Abhandlungen veröffentlicht worden ist, in Kürze zusammenzufassen.

Fall 1. Georg la Barre aus Halberstadt, 12 Jahre alt, kam im Juli 1875 in die Klinik des Prof. Graefe in Halle wegen einer Geschwulst, die das obere Lid des linken Auges und den an dasselbe anstossenden Theil der Schläfengegend einnahm. Die Geschwulst hatte sich, wie die Anamnese ergab, sehr langsam entwickelt und soll schon, als der Knabe 6 Monate alt war, bemerkt worden sein. Sie soll damals etwa die Grösse eines gewöhnlichen Gerstenkorns gehabt hahen. $0 \mathrm{~b}$ bei der Geburt schon Anfänge da waren, lässt sich nicht mehr feststellen. In der Familie des Kranken sind derartige oder ähnliche Geschwulstbildungen, soweit sich ermitteln lässt, nicht vorgekommen. Eine spontane Schmerzbaftigkeit der Geschwulst hat nie bestanden. Der Knabe ist äbrigens gat entwickelt und zeigt gute geistige Fabigkeiten.

$\mathrm{Zu}$ Ostern 1874 wurden von einem in Halberstadt ansässigen Arzt zwei bohnengrosse Stücke aus der Geschwulst excidirt. Jedoch soll sie an derselben Stelle wiedergekebrt sein. In den letzten vier Wochen begann sie stärker zu wachsen. Es bestand seitdem starke Ptosis. Wenn der Patient Schnupfen hatte, war die Lidspalte selbst völlig geschlossen.

Als der Kranke in die Klinjk gebracht wurde, hing das obere Lid sackähnlich über die Lldspalte hinab, so dass das Sehen mit dem betreffenden Auge fast unmöglich war. Die Consistenz der Geschwulst war teigig; in der Tiefe liessen sich 
Dirchows Irchiv BdLLX.
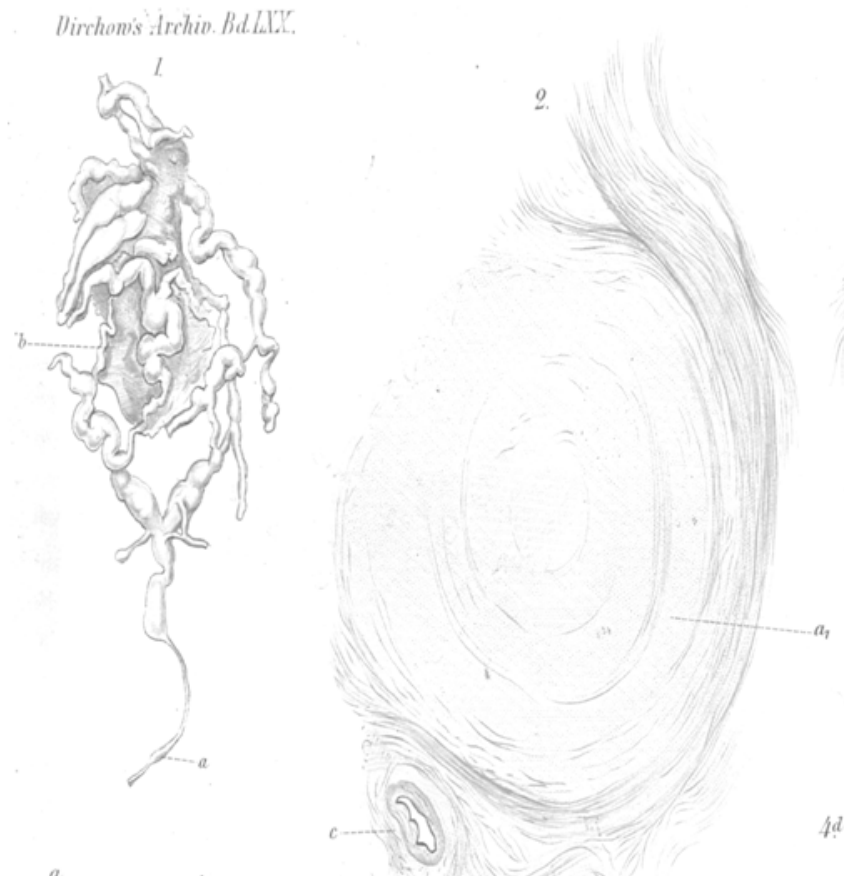

Taf.I.
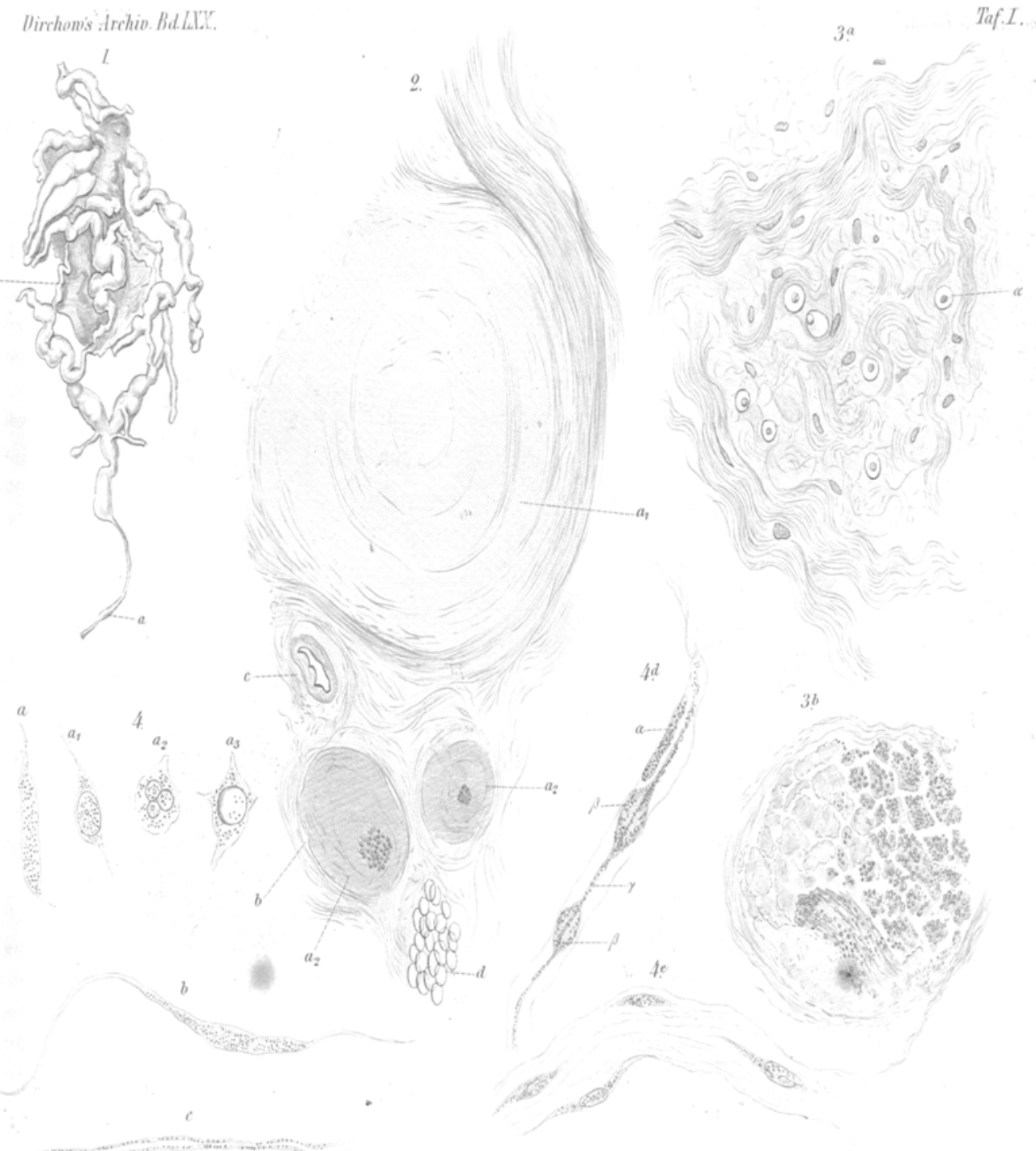
einzelne resistentere Knoten und Stränge deutlich durchfühlen. Stärkerer Druck auf dieselben war schmerzhaft. Die Haut des Lides wie des Schläfentheiles der Geschwulst zeigte äbrigens normale Bescbalfenheit. Es wurde die Diagnose auf ein „Neuroma plexiforme" gestellt; doch konnte ein Lymphangiom mit Sicherheit nicht ausgeschlossen werden.

Die von Prof. Graefe am 24. Juli vorgenommene Exstirpation war ziemlich schwierig, da die Geschwulst sich tief in die Orbita hinein, unter dem Dach derselben fortsetzte. Es wurde ein Schnitt längs des Tarsus äber den Augenwinkel hinweg bis auf die Schläfe geführt, das Lig. tarsi superioris durchschnitten und ein Theil des $M$. réctus superior excidirt, der von der Geschwolstmasse umgeben war. Schliesslich wurden die Reste der Geschwulst mit dem scharfen Löffel fortgenommen. Die an der Schläfe gelegene Partie des Tumors wurde unter Schonung der bedeckenden Haut entfernt. Die Operation verlief im Ganzen gut. Die Heilung erfolgte per secundam intentionem, obne wesentliche Störungen.

Zum Behuf der besseren Uebersicht möge bier gleich der zweite, zu meiner Beobachtung gekommene fall angeschlossen werden; die anatomische Untersuchung beider folgt unten im $\mathrm{Zu}$ sammenhange.

Fall 2. August Bullert, 8 Jabre alt, Schlossersohn aus Halberstadt, kam am 13. Juli 1875 in die Klinik des Prof. Volkmann in Halle wegen einer Geschwulst am Kopfe. Durch die Anamnese wurde festgestellt, dass der Kleine früher durchaus gesund gewesen war. Erst vor 4 Jahren wurde über dem rechten Ohre eine kleine Geschwulst bemerkt, die seitdem stetig anwuchs. Die Haut, welche die Geschwalst bedeckte, war stets von normaler Beschaffenheit. Die Geschwulst wuchs obne irgendwelche Beschwerden für den Kranken flach über die Schuppe des Schläfenbeines fort bis auf das Scheitelbein.

Am 11. December 1874 wurde eine Incision in die Geschwulst gemacht und unter mässiger Blutung ein keilförmiges Stäck excidirt. Aus der mikroskopischen Untersuchung wurde damals auf Leontiasis geschlossen.

Als der Knabe am 13. Juli $187 \mathfrak{3}$ in die Klinik kam, bestand eine weiche, schlaffe, wenig druckempfindliche Geschwulst, die sich von der recbten Hinterohrgegend über Schläfenschuppe und Scheitelbein ziemlich bis zur Sagittalnaht erstreckte. Sie bedeckte eine Fläche, die in der grössten Länge 5, in der grössten Breite $4 \frac{1}{2}$ Zoll maass. Sie überragte das umliegende Hauptniveau an der dicksten Stelle etwa um einen Zoll. Die Haut, welche die Geschwulst bedeckte, zeigte sich unverändert. Bei energischer Palpation fühlte man die schlaffen Gewebsmassen der Geschwulst von harten, mit kJeinen Knötchen durchsetzten Strängen durchzogen. Die Diagnose wurde auf ein plexiformes Neurom des Kopfes gestellt.

Die Operation der Geschwulst wurde unter allen Cautelen der antiseptiscben Methode ausgeführt. Es wurden zunächst parallel zur Pfeilnaht zwei 3 Zoll lange, $1 \frac{1}{2}$ Zoll breite Stücke der Geschwulstmasse so excidirt, dass zwischen beiden eine 1 Zoll breite Brücke übrig blieb. Dabei trat eine ziemlich starke Blutung aus grösseren Arterien auf. Die Geschwulst wurde bis zum Periost fortgenommen, 
die Rănder weithin unterminir, ebenso wie die Brücke. Die entstandenen Defecte wurden bis auf einen halben Zoll breite Spalten durch Spannnähte geschlossen. Der Verband wurde nach der Lister'schen Methade angelegt. Die gleich nach der Operation vorgenommene Untersuchung bestätigte die vorhergestellte Diagnose, indem sich in den excidirten Stücken die weichen Geschwulstmassen von varikös verdickten Nervensträngen dicht durchzogen zeigten. Der Wundverlauf war durchaus günstig: die unterminirten Ränder verklebten mit der Unterlage, ohne dass Eiterung eintrat. Die Wundränder blieben reactionslos. Schmerzen traten nicht auf. Die Abendtemperaturen schwankten in der ersten Woche zwischen 38,0 und $38,8^{\circ}$. Dann blieben sie normal. Am 24. Juli wurde zu einem Verband mit Benzoëwatte übergegangen. Am 29. Juli wurde eine nochmalige Excision gemacht, indem die Schnitte diesmal vom Tuber parietale gerade nach hinten in einer Länge von 3 Zollen geführt wurden. Während der ersten 4 Tage wurde der Lister'sche Verband angewendet. Dabei stieg die Abendtemperatur am 2. Tage auf $39,2^{\circ}$, sank dann aber wieder, so dass am 5. Tage zum Verbande mit Benzoëwatte übergegangen werden konnte. Da die Granulationen der ersten Wunde sehr schlaff gewesen waren, wurde die Wunde am 29. Juli mit dem scharfen Löffel behandelt. Am 9. August wurde der Knabe mit der Weisung, noch einige Zeit Ueberschläge von Arg. nitr. zu machen, nach Haus entlassen.

Da noch Reste der Geschwulst zurückgeblieben sind, die voraussichtlich stetig fortwachsen werden, so wird jedenfalls mindestens eine nochmalige Operation nöthig werden, ehe an voliständige Beseitigung der Geschwulst zu denken ist.

\section{Anatomische Untersuchung yon Fall 1.}

Die Geschwulstmasse war in 2 Stücken entfernt worden, die wesentlich dasselbe Verhalten zeigten. Ek̉ne lockere Bindegewebsmasse, in die ein Haufe von vielfach gewundenen, bald dickeren, bald dünneren Strängen von röthlichweisser Farbe und viel bedeutenderer Consistenz, als sie das umgebende Gewebe hatte, eingebettet war. Die Stränge zeigten sich stellenweise verzweigt und durch Anastomosen mit einander verbunden. An mehreren Stellen war der Uebergang derselben in einen feinen Nervenfaden zu constatiren.

Als Verfasser das Präparat zur Untersuchung erhielt, hatte es bereits einge Wochen in Müller'scher Fü̈ssigkeit, dann in Spiritus gelegen und war durch diese Agentien in der bekannten Weise verăndert worden.

Nachdem das eine Stück des Präparates von dem umbüllenden Gewebe befreit war, was ohne besondere Mühe gelang, fanden sich die bereits genannten, im Ganzen cylindrischen Stränge von sebr ungleichmässiger Dicke, stellenweise zu rundlichen Knoten angeschwollen, mannichfach gewunden, hin und wieder verzweigt oder anastomosirend. (S. Fig. 1.)

Behufs genauerer Untersuchung wurden zunächst Querschnitte von den sehr zähen, fibrösen Strängen angefertigt. Auf diesen Durchschnitten erkannte man im Centrum der dünneren eines dunkleren Punkt, der, wie sich bei stärkeren Vergrösserungen ergab, aus durcbschnittenen Nervenfasern bestand. Die stärkeren Stränge - sie erreichten bei diesem Präparate einen Durchmesser von etwa 3-4 Mm, zeigten sich auf dem Querschnitt ganz homogen und gelblichweiss gefärbt. 
Bei mässigen Vergrösserungen konnte man bemerken, dass die Peripherie der Stränge aus concentrisch angeordueten Schichten bestand, die etwa den vierten Theil des Gesammtdurchmessers einnahmen. Centralwärts von diesen befand sich ein mehr bomogener Bezirk, in welchem bei den dünneren Strängen ein rundlicher Haufe von dunklen Punkten, den Querschnitten ebensovieler Nervenfasern entsprechend, sich vorfand. Die Querschnitte der Siränge zeigten sich von schmalen Spaltrâumen umgeben, die, wie sich später ergab, als die perinenralen Lymphrăume zu betrachten sind, die trotz der Geschwulstbildung erhalten blieben. Zwischen den Strängen fanden sich hin und wieder die Querschnitte wohlerhaltener Nervenstämmchen ohne alle Abnormitäten. (S. Fig. 2.)

Bei Anwendung stärkerer Vergrösserungen (Hartnack 0bj. Vll. Oc. 3) ergab sich, dass die Stränge ihrer Hauptmasse nach aus faserigem Bindegewebe bestanden. Die concentrischen Schichten der Peripherie zeigten derbe circulär verlaufende Faserbündel. Nach innen von diesen waren vielfach geschlängelte Faserzüge sichtbar, dem Anscheine nach bedeutend Iockerer, als die ersteren. Diese Fasern waren sehr unregelmässig angeordnet und vielfach untereinander verschlungen und verwebt. In der Nähe der meist central, öfters anch etwas excentrisch gelegenen Nervenfasern - wo dieselben vorhanden waren - nahmen die Bindegewebszüge eine mebr zu diesen parallele Richtung an, z. Th. aber schlangen sie sich in mannichfachen Windungen zwischen den Nervenfasern hindurch. An Strängen mittlerer Dicke zeigten die Nervenfasern keine bestimmte Anordnung mebr, sondern waren zwischen reichlichen Bindegewebsmassen einzeln verstreut sichtbar. Bei der Behandlung mit carminsaurem Ammoniak wurde der ganze Bau der Geschwulstmassen deutlicher. Es traten jetzt zahireiche dunkle Kerne zwischen den Bindegewebsfasern hervor, von meist längsovaler Gestalt, parallel zu diesen gerichtet. Stellenweise waren sie im Querschnitt getroffen und erschienen dann als rundliche rothe Scheibchen. Die Markscheide der Nervenfasern - nur markhaltige waren deutlich sichtbar - hob sich von dem dunbelgefärbten Axencylinder bestimmt ab; die Schwann'sche Scheide war deutlich zu erkennen. Alle diese Bestandtheile erschienen bei manchen Fasern, trotzdem sie von gewaltigen Bindegewehsmassen dicht umgeben waren, ziemlich unverändert. Noch deutlicher war das ganze Bild an Präparaten, die mit Picrocarmin gefärbt waren: (S. Fig. 3 a.)

Das zwiscben den Strängen gelegene Gewebe zeigte einen locker fibrillären Bau. An einzelnen Stellen fanden sich darin Häufchen von Fettzellen. Kleinere Vesen und Arterien waren nicht selten im Querschnitt getroffen. Auch sab man an manchen Querschnitten zabireiche kleine Gefässchen, besonders Capillaren, in den verschiedensten Richtungen durch die Stränge verlaufen. Stellenweise war die Zahl der Kerne in der Wandung der Capillaren vermehrt, auch die Wandung mancher grösseren Gefässe schien die normale Stärke zu überschreiten. In den Spalträumen, die die Stränge ungaben, liess sich eine Endothelmembran constatiren, die sich als zartes, granulirtes Häutchen mit ovalen, ziemlich gleichmässig weit von einander entfernten Kernen von der Wand abgelöst hatte. Zahlreiche Querschnitte, die durch alle möglichen Stellen der Geschwulst gemacht wurden, zeigten genau das nehmliche Verhalten.

Wie bereits oben erwähnt wurde, fanden sich einzelne feine Nervenstämmchen 
in der Geschwulst, die allmählich zn immer starker werdenden Străngen anschwollen. An diesen musste sich das allmähliche Auftreten der Geschwulstbildung am besten studiren lassen. Es wurden zu dem Behufe von einer Strecke von einigen Millimetern, die den Anfang der Verdickung zelgte, 28 Querschnitte gemacht, die dies Nervenstückchen in nahezu continuirliche Abschnitte zerlegten.

Der erste Querschnitt batte einen Durchmesser von etwa $\frac{1}{2} \mathrm{Mm}$, der letzte einen solchen von $1 \frac{1}{2} \mathrm{Mm}$. Der Querschnitt blieb im Ganzen ziemlich kreisförmig. Während nun der erste Querschnitt dem eines völlig normalen Nerven glich, zeigte sich bereits an den vächsten Schnitten eine deutliche Veränderung, die im Ganzen in einer Vermehrung des Perineurium externum und internum bestand, im Einzelnen jedoch derartig war, dass die secundären und tertiären Bündel des Stranges im Ganzen gewahrt blieben, die Nervenfasern aber in einigen derselben vollständig, in anderen nur z. Th. schwanden und durch Bindegewebe ersetzt wurden, während noch andere Bündel, die meist ziemlich central gelegen waren, im Ganzen intact blieben. Allenthalben waren zwischen den Bündeln, auch wenn sie ganz von Nervenfasern frei waren, die dieselben umgebenden Lymphräume deutlich. (S. Fig. 3 b.)

In der Peripherie, dem Perineurium externum entsprechend, zeigte sich gröberes circulär angeordnetes Bindegewebe, das den ganzen Stamm umhüllte. Zugleich mit der Bindegewebsentwickelung wurden zahlreiche Kerne sichıbar. In den späteren Schnitten verlor sich der lappige Bau des Quersehnittes mehr und mehr; es traten dagegen zahllose Faserzüge auf, die sich in allen Richtungen durcbsetzten. Die concentrische Schichtung der Peripherie wurde immer massiger. Die Nervenfasern zerstreuten sich dabei bald, bald traten sie wieder näher zusammen. Im Ganzen war es deutlich, dass sie an Zahl abgenommen hatten. - In manchen von den stärkeren und in den stärksten Strängen war von Nervenfasern gar nichts mehr zu sehen. In einigen von diesen traten dagegen zahlreiche Capillaren auf. An einer Stelle der Geschwulst fanden sich, ganz umgeben von Geschwulststrängen, Muskelfasern vor, die vom M. rectus superior stammten; sie zeigten völlig normale Beschaffenheit.

Um die Veränderungen in ibrem feineren Detail kennen zu lernen, wurden jetzt Zerfaserungspräparate gemacht. Diese Methode stiess bei der grossen Zähigkeit der Stränge auf einige Schwierigkeiten; doch gelang es, Bindegewebs - und Nervenfasern, wie auch Gefässe, auf Strecken zu isoliren. Zur Untersuchung wurde Hartnack 0 bj. X, à Immers. und 0 c. 3 verwendet.

Es fanden sich in mehreren dünnen Strängen, die die Veränderung erst im Begion zeigten, besonders massenhaft Bindegewebsfibrillen, die in welligen Zügen angeordnet waren. In diesen Zügen liessen sich zahlreiche spindelförmige Elemente erkennen, die auch isolirt erhalten wurden. (S. Fig. 4 e, b und a, $a_{1}, a_{2}, a_{3}$.) Sie liefen oft in lange, feine Fortsätze aus, deren Endigung sich nicht verfolgen liess. Auch fanden sich zahlrelche längsovale Kerne zwischen den Bindegewebsfibrillen.

Kleine Arterien, Venen und Capillaren liessen sich leicht darstellen. Sie erschienen z. Th. mit Blutkörperchen gefültt, z. Th. leer. (Aeltere Thromben lieșsen sich nirgends nachweisen.) Die Beschaffenheit der Wandung der Gefässe sclien vom Normalen nirgends wesentlich abzuweichen.

Die sehr leicht und in Menge nachzuweisenden Nervenfasern zeigten sämmtlich 
eine deutliche Schwann'sche Scheide. Die Markscheide fand sich auf allen Stufen der Gerinnung resp. der Atrophie. Je nach der Menge des Markes wechselte die Dicke der Fasern. Einige zeigten körnige Markmassen, stellenweise spindelförmig angehäuft, während die Schwann'sche Scheide an den markarmen Stellen deutlich als intacte Membran mit einzelnen Kernen sichtbar war. (S. Fig. 4c, d.) Somit liess sich auch an den Nervenfasern eine wesentliche Veränderung, mit Ausnahme der retrograden (Atrophie), nicht nachweisen.

\section{Anatomische Untersuchung von Fall 2.}

Der Bau der Geschwulst wich nur in ziemlich unwesentlichen Punkten von dem bei Fall 1 besprochenen ab, so dass hier nur noch wenig zu dem oben Gesagten hinzuzufügen ist:

Die ganze Geschwulst hatte in diesem Falle grössere Dimensionen. Dem entsprechend waren die Stränge, die ebenso wie bei Fall 1 eine zusammenhängende Masse von mannichfach gewundenen Gebilden von bald cylindrischer, bald mehr abgeplatteter Form, mit sehr wechselndem Durchmesser, darstellten, bedeutend stärker entwickelt. Ihre Dicke stieg bis auf $1 \mathrm{Cm}$. Sie lagen zum grössten Theile im Unterhautzellgewebe. Zuweilen zeigten sie stumpfe kolbige Enden (deren feine Fortsätze wabrscheinlich abgerissen waren); an anderen Stellen liefen sie in feine Fäden aus. Die Geschwulstmasse bing im Ganzen continuirlich mit der Cutis zusammen.

Die Haut selbst, die diesem Präparate z. Th. noch anhing, erschien wenig verändert: die Epidermis war von normaler Beschaffenheit, die Cutis etwas verdickt, mit merklicher Vermehrung des Bindegewehes; übrigens waren Abnormitäten nicht 31 bemerken. Das Unterhautzellgewebe zeigte die Beschaffenheit eines lockeren, wenig fetthaltigen Bindegewebes, das an Masse beträchtlich vermehrt war. In dieses Gewebe waren die genannten Stränge eingebettet.

Auch von diesem Präparat wurden zunächst Querschnitte angefertigt. So wurde auch hier eine Anzahl von continuirlichen Schnitten von einem feinen Strange gemacht, der allmählich stärker wurde. Der Anfang desselben zeigte einen Durchmesser von $1 \mathrm{Mm}$., während der letzte Querdurchschnitt $4 \mathrm{Mm}$. Durchmesser besass. Auch hier liess sich die successive Abnahme der Nervenfasern deutlich constatiren. Anch die Lymphräume, welche die Stränge umgaben, wurden nachgewiesen. - Zerfaserungspräparate, die ebenfalls angefertigt wurden, ergaben genau dieselben Elemente, wie bei Fall 1: Das an Spindelzellen reiche, faserige Bindegewebe machte auch hier die Hauptmasse der Geschwulst aus; dazwischen fanden sich Arterien, Venen und Capillaren von normaler Beschaffenheit. Nervenfasern, welche, da grössere Knoten zur Zerfaserung benutzt wurden, nicht bäufig aufzufinden waren, zeigten eine normale Schwann'sche Scheide, die Markscheide in allen Stadien der Verfettung und Atrophie.

Zusammenstellung der aus der Literatur bekannten Fälle.

Fall 1. Der erste bekannte Fall von plexiformem Neurom ist der von Depaul und Verneuil') im Jahre 1857 veröffentlichte.

1) S. Bulletins de la société anatomique de Paris. XXXII. Année; 1857. 2. Série. Tome II, p. 24. 
Er betrifft einen jungen Mann, der ein plexiformes Neurom am Nacken (N. occipitalis maj. ?) und ein solches des ganzen linken Plexus cervicalis besass. Die näheren Details, besonders auch die anatomische Untersuchung finden sich in der angegebenen Abhandlung.

Fall 2. Von Lotzbeck im Jahre 1858 veröffentlicht ${ }^{\mathrm{J}}$ ). Derselbe findet sich auch genau beschrieben bei P. Bruns ${ }^{2}$ ).

Angeborenes plexiformes Neurom der Lendenwirbel- und Kreazbeingegend mit gleichzeitiger Rückgratsspalte. - Lotzbeck fasste die Geschwulst nach der mikroskopischen Untersuchung als reines Fibroid auf. Nach der Untersuchung von P. Bruns (am Spirituspräparat im Jahre 1870) war ibr Bau ganz wie bei dem von ihm selbst veröffentlichten Falle.

Fall 3. Aus dem Jahre 1861. Von Verneuil veröffentlicht ${ }^{3}$ ). In diesem Falle bestand ein wahrscheinlich erworbenes plexiformes Neurom des Saumes der Vorhaut, welches sich unter chronisch entzündlichen Erscheinungen entwickelt hatte und von einer enormen Hyperästhesie der befallenen Stelle begleitet war. Verneuil wählte für die Geschwulst den Namen "Neuroma cylindricum plexiforme“. Er erwähnt auch, dass zwei Fälle vom Plexus cervicalis in den „0bservations inédites de M. M. Depaul et Guersant ${ }^{4}$ " enthalten seien.

Fall $4^{5}$ ). Ebenso wie Fall 5 von Billroth beobachtet. Er stammt aus dem Jahre 1863.

Plexiformes Neurom des rechten oberen Augenlides and des benachbarten Schläfentheiles.

Fall $\left.5^{6}\right)$. Aus dem Jahre 1869.

Plexiformes Neurom des linken oberen Lides, nebst der entsprechenden Schläfenpartie.

Die nächsten 3 zur Beobachtung gekommenen Fälle wurden von P. Bruns ${ }^{7}$ ) beschrieben. (1870.)

Fall 6. Angebornes plexiformes Neurom über dem rechten 0hre nebst den benachbarten Partien des Kopfes.

Fall 7. Angebornes plexifurmes Neurom des linken oberen Lides und der linken Schläfengegend neben gleicbzeitiger allgemeiner Neuromatose.

Fall 8. Angebornes plexiformes Neurom des linken oberen Lides und der linken Schläfengegend.

Fall $9^{8}$ ). Dieser von Czerny im Jabre 1873 publicirte Fall zeigt eine Combination von Elephantiasis Arabum congenita mit plexiformen Neuromen. Eine sehr- grosse, elephantiastische Geschwulst bestand hier in der Lumbal- und Sacralgegend, gleichzeitig mit plexiformen Neuromen aller vorderen und hinteren Aeste

1) S. Lotzbeck, Dje angebornen Geschwülste der hinteren Kreuzbeingegend. Inaugualdissert. München 1858. S. 18. Fig. 3-6.

2) P. Bruns, Das Rankenneurom. Dieses Archiv Bd. 50. 1870. Heft 1. S. 80.

3) Archives générales de médicine. 1861 . Vol. II. V. Série. Tome 18. p. 537.

4) - Diese konnte ich mir nicht verschaffen.

5) S. Langenbeck's Archiv. Bd. IV. 1863. S. 545.

6) S. Langenbeck's Archiv. Bd. XI. 1869. S. 230.

7) S. P. Bruns, a. a. 0 .

8) S. Gzerny, Langenbeck's Archiv, Bd. XVIL. No. 15. S. 357. 
der Lumbalnerven and der hinteren Aeste der Sacralnerven. Auch der Lumbaltheil des $\mathrm{N}$. sympatbicus war betroffen.

Fall $10^{1}$ ). Plexiformes Neurom des Plexus brachialis. Gleichzeitige Sarcom. bildung. (1873.)

Soweit sich nach den oben angegebenen 12 Fällen (incl. der 2 von mir beschriebenen) eine Charakteristik der vorliegenden Geschwulstform geben lässt, will ich dies auf den folgenden Seiten versuchen; mit dem Ruickhalt freilich, dass zwar die Symptomatologie der. Geschwulst sich in einer lür die practische Medicin ziemlich ausreichenden Weise geben lässt, dagegen namentlich in Bezug auf die feinere Anatomie noch weitere Untersuchungen sehr wünschenswerth erscheinen.

\section{Aetiologie.}

Man kann nicht sagen, dass das plexiforme Neurom in ausgesprochener Weise hereditär wäre. In den wenigen Fällen, wo eine Vererbung von Geschwülsten vorkam, betraf dieselbe wohl mehr die häufig zugleich vorkommende elephantiastische Form der Geschwulstbildung, als die plexiforme Neurombildung selbst. In 10 von den obigen 12 Fällen ist gar keine Heredität nachzuweisen. In den übrigen betraf die Erkrankung einmal 2 Brüder in fast genau gleicher Form, nur dass einer derselben noch an allgemeiner Neuromatose litt (s. Lit. Fall 7). Die Mutter dieser beiden litt vielleicht ebenfalls an einer Neuromatose (Haulgeschwülste). In einem anderen Falle (s. Lit. Fall 9) ging eine Geschwulstkrankbeit durch 4 Generationen einer Familie hindurch. Doch ist erst bei der letzten (Therese Geng) das Auftreten von plexiformen Neuromen erwiesen. lhre Mutter (Rosine Geng) besass wenigstens in der exstirpirten Geschwulst ${ }^{2}$, die übrigens der der Tochter ähnlich war, deren keine. In 2 Fällen bestand neben den plexiformen Neuromen gleichzeitig ausgebreitete allgemeine Neuromatose. Im Uebrigen befielen dieselben meist ganz normal entwickelte Individuen.

Die Zeit der Entwickelung der Geschwulst fällt jedenfalls in vielen, vielleicht den meisten der beobachteten Fälle, in die Zeit des Fötallebens. Sicher angeboren war sie in 5 von den obigen

1) S. A. von Winiwarter, Langenbeck's Archiv. Bd. XIX. S. ว95.

2) Beschrieben bei Fr. Hecker, Die Elephantiasis oder Lepra arabica, Lahr 1858. 
Fällen (Lit. Fall 2, 6, 7, 8, 9); in 7 Fällen ist dies zweifelhalt, in 4 Fällen, wo sie bereits in den ersten Lebensjahren bemerkt wurde, wahrscheinlich (s. mein Fall 1, 2; Lit. Fall 5,11), in den anderen nicht mit Sicherheit abzuweisen; nur in dem Verneuil'schen Falle (Lit. Fall 3) scheint die Geschwulst erworben zu sejn. In dem Czerny'schen Falle war die Geschwulst der Kreuzbeingegend angeboren; über die plexiformen Neurome selbst fehlt eine bestimmte Angabe.

Dem Sitz nach scheint die Geschwulst gewisse Nervenbezirke entschieden $z u$ bevorzugen. Denn in 5 von 12 Fällen fand sie sich in der Gegend des oberen Augenlides und erstreckte sich dann regelmässig auf den anstossenden Theil der Schläfe, zuweilen (2 Fälle) auch auf die Gegend der Augenbrauen resp. den unteren Theil derselben Stirnseite. In diesen Fällen drang sie wohl stets tief in die Orbitalhöhle unter dem Dach derselben ein, was in 2 Fällen besonders angeführt ist (s. mein Fall 1 und Lit. Fall 5). Nächst den genannten Stellen scheint die Geschwulstbildung die Gegend des Schuppentheiles des Schläfenbeins mit Vorliebe zu befallen, von wo sie auf benachbarte Regionen des Kopfes sich verbreitete, so z. B. in meinem Fall 2 bis nahe an die Sagittalnaht fortschritt. (Alle diese Fälle betreffen das Gebiet der Ausbreitung des N. trigeminus; vielleicht noch einiger anderen Nerven.) In 2 Fällen wurden plexiforme Neurome in der Lendenwirbel- und Kreuzbeingegend beobachtet, freilich beschränkte sich die Geschwulst nur in einem Falle auf diese eine Stelle (Lit. Fall 2), während in dem anderen (Lit. Fall 9) fast sämmtliche Lendennerven, vordere und hintere Aeste, sowie die hinteren Aeste der Kreuzbeinnerven, befallen waren. Auch der Lendentheil des N. sympathicus, besonders die zu den Gerässen gehenden Zweige desselben, zeigten sich hier mitbetroffen. (Czerny nimmt selbst an, dass nur die sympathischen Fasern, welche mit den grossen Nervenstämmen verlaufen, von der Erkrankung betroffen seien.) In einem Falle (Lit. Fall 3) wurde die Geschwulst am Saume des Präputium beobachtet. In einem anderen war neben den Nerven des behaarten Theiles der Nackengegend (vielleicht $\mathrm{N}$. occipitalis $\mathrm{mj}$.) der ganze Plexus cervicalis befallen (Lit. Fall 1. Auch sind 2 weitere Fälle vom Pl. cervic. bei Fall 3 angeführt). Einmal beschränkte sich die Ausbreitung der Geschwulst auf den Plexus brachialis (Lit. Fall 10): 
Symptomatologie.

Die plexiformen Neurome wachsen im Ganzen langsam und stetig. Zuweilen fingen sie nach einigen Jahren langsamen Wachsthums an, sich schneller zu vergrössern. Immer erstreckte sich ihr Wachsthum über viele Jahre. So bestand die Geschwulst in einem Falle (Lit. Fall 7) während 33 Jahren, ein anderer Fall von angeborenem plexiformen Neurom kam erst im 43. Lebensjahre des Patienten zur Beobachtung (Lit. Fall 8). In einigen Fällen findet sich die Angabe, dass die Geschwulst in derselben Ausdehnung, in der sie angeboren war, in die Höhe gewachsen sei, obne an Flächenausdehnung zuzunehmen. Für diese Fälle wäre anzunehmen, dass ein bestimmter Bezirk von Anfang an befallen war und in derselben Ausdehnung auch befallen blieb. Für solche Fälle wäre natürlich die Prognose der Operation in Bezug auf etwaige Recidive sehr günstig zu stellen (die Angabe findet sich z. B. bei Lit. Fall 6). In einigen Fällen scheint übrigens doch eine flächenhafte Vergrösserung angenommen werden zu müssen (so bei meinem Fall 2).

Die Grösse der durch die plexiformen Neurome veranlassten Geschwülste ist ausserordentlich verschieden. In dem von Verneuil beobachteten Falle war die Vergrösserung des befallenen Präputium zwar beträchtlich, aber doch mit der durch andere Geschwülste veranlassten nicht zu vergleichen, während in dem von Czerny beobachteten Falle die Geschwulstbildung eine colossale Ausdehnung annahm, wenn auch die auf dem Rücken jener Patientin befindliche grosse Geschwulst wohl wesentlich auf Rechnung der gleichzeitigen Elephantiasis zu schieben ist. In manchen Fällen waren ganze grosse Nervengebiete befallen, wie z. B. in eben jenem letzterwähnten Falle, in anderen nur ein sehr. beschränkter Theil einer Nervenausbreitung.

Die äussere Form dieser Geschwülste ist gleichfalls sehr verschieden, doch ist diese Verschiedenheit mehr durch ihre Ausdehnung und den Grad ihrer Entwickelung bedingt, als dass sie wesentliche Eigenthümlickeiten der Form beträfe. Im Ganzen findet sich die Haut an der Stelle der Geschwulstbildung in Form einer oder mehrerer verschieden mächtiger Falten oder Wülste erhoben, wenn dieselbe die in der Haut oder im Unterhautzellgewebe ver laufenden Nerven befallen hatte, während diese Vorwölbung weniger 
deutlich ist in Fällen, wo tiefer liegende Nerven betroffen sínd; doch pflegte die Bildung von Wulstungen und Prominenzen der Haut nicht zu fehlen, da meist die oberflächlichen Nerven, wenigstens zum Theil, ebenfalls befallen waren. Nur wo die elephantiastische Umbildung des Unterhautzellgewebes sehr beträchtlich war, trat an die Stelle der Faltenbildung ein mehr massiger Tumor von verschiedener Form.

In den meisten der beschriebenen Fälle war die Consistenz im Ganzen als teigig-weich zu bezeichnen (wie die eines Molluscum fibrosum, Billroth). In wenigen Fällen, wo die Zwischenmasse eine dichtere Beschaffenheit besass, bot die Geschwulst auch für den tastenden Finger eine grössere Resistenz dar. War die Grundmasse nicht zu derb, so liessen sich bei tieferer Palpation stets bald dickere, bald dünnere wurmartig gewundene Stränge, von mehr oder weniger zahlreichen knotigen Anschwellungen unterbrochen, hindurchfüblen. Diese besassen immer eine viel beträchtlichere Festigkeit als das umgebende Gewebe und liessen sich zuweilen in der weicheren Umgebung merklich verschieben. Verneuil verglich diese Stränge den Wülsten einer Varicocele.

Die die Geschwulst bedeckende Haut bot in éinigen Fällen keine merkliche Veränderung dar, in anderen konnte man füblen, dass sie verdickt war. Zuweilen liess sie sich nicht mehr in einer Falte aufheben oder war selbst völlig unverschieblich. In mehreren Fällen fanden sich abnorme, bräunlicke oder selbst rostbraune Pigmentirungen derselben. Auch war sie bisweilen mit auffallend starken, krausen Haaren besetzt. (Eine Pigmentirung wurde bisher am Kopfe nicht beobachtet.) In 2 Fällen fand sie sich in eczematösem Zustande.

Von subjectiven Symptomen ist nur anzuführen, dass bei Druck auf die Geschwülste, besonders auf die in der Tiefe gelegenen Knoten und Stränge, Schmerzen auftraten, die bisweilen eine ausserordentliche Höhe erreichten. In einigen Fällen fehlten dieselben. Meist bestanden spontan keine Schmerzen. In einem Falle wurden stechende Schmerzen in der Geschwulst gefühlt (Lit. Fall 2), in einem anderen fast continuirliche, in die Umgebung ausstrablende Schmerzen (Lit. Fall 10), in einem Falle endlich ist eine besondere Empfindlichkeit gegen stärkere Temperaturdifferenzen angegeben (Lit. Fall 6), Die in dem Verneuil'schen Falle bestehende hoch- 
gradige Hyperästhesie der Vorhaut ist vielleicht zum Theil den chronisch-entzündlichen Zuständen derselben zuzuschreiben, die erst secundär die Veränderung an den Nerven zur Folge hatten.

Die durch die Geschwulst veranlassten localen Störungen können ziemlich hohe Grade erreichen: So ging in zwei Fällen in Folge der zunehmenden Geschwulstentwickelung im oberen Augenlid der Bulbus zu Grunde; durch den Druck der intraorbitalen Massen kam eine Vergrösserung der Orbita zu Stande, deren Knochen gleichzeitig an der Hypertrophie des umliegenden Gewebes Theil nahmen; Vergrösserungen der Intervertebrallöcher, durch welche verdickte Nervenstämme austreten, wurden beobachtet; eirmal zeigte sich selbst der Schädel in Folge des Druckes einer Geschwulstpartie durchbrochen, so dass ein kreisrunder Defect von 1,5 Cm. Durchmesser entstanden war (Lit. Fall 6). In einem anderen Falle kam durch die weit vorgeschrittene Entariung der Nervenstämme (oder durch die gleichzeitige Sarcombildung?) Paralyse der betroffenen Nervengebiete zu Stande (Lit. Fall 10).

Das Allgemeinbefiaden litt in keinem der beobachteten Fälle in merklicher Weise, ausser in den durch Sarcombildung oder colossale Grade von Elephantiasis complicirten.

Eigentliche Recidive sind bisher nicht beobachtet (s. auch das oben über das Wachsthum der plexiformen Neurome Gesagte). Somit wäre die Prognose der Geschwülste im Ganzen durchaus günstig zu stellen. Die Diagnose wird sich nach den oben angegebenen Symptomen nicht immer mit voller Sicherheit stellen lassen, da man, wenn für die Natur der Stränge als verdickter Nerven kein Anhaltspunkt durch die Lage oder den Druckschmerz gegeben ist, dieselben wohl mil thrombosirten Venen- oder Lymphgefässectasien verwechseln könnte. Daher wurde denn auch in einem Falle die Diagnose auf ,angeborenes Lipom mit cavernöser Venenectasie" gestellt.

Die Behandlung der Geschwulst, falls dieselbe überhaupt eingeleitet werden soll, kann nur in der Operation bestehen. Bei den ausgebreiteteren Formen empfiehlt sich die Methode der mehrfach wiederholten partiellen Excisionen mit theilweiser Schonung der Haut, wie dieselbe in unserem Falle 2 von Prof. Volkmann angewendet wurde. - Bei geringerer Ausdehnung wird sich meist die Entfernung durch einmalige Operation bewerkstelligen lassen, 
doch war dieselbe in manchen Fällen, wo die Strangbildungen z. B. tief in die Orbitalhöhle eindrangen, nicht leicht. In unserem Fall 1 musste der scharfe Löffel zu Hülfe genommen werden, um die Reste der Geschwulst völlig zu entfernen. Die bei weithin ausgedehnten Erkrankungen nöthige Unterminirung der Hautränder gebietet jedenfalls besondere Vorsicht. In einem Falle wurde ein solcher Hautlappen brandig und es erfolgte Tod an Pyämie. In den leichteren Fällen, die das obere Augenlid und die Schläfengegend betrafen, war der Ausgang meist günstig.

\section{Pathologisch-Anatomisches und Entwickelung der Geschwulst.}

\section{Gröbere Anatomie.}

Das Grundgewebe, in welches die Geschwulststränge eingebettet waren, bestand in fast allen Fäilen aus einer Bindegewebsmasse von geringer Consistenz, die bei der Lage der Geschwulst im Unterhautzellgewebe mit der Cutis innig zusammenhing. In einigen Fällen war diese Zwischensubstanz zwischen den Strängen von fast schlejmiger Beschaffenheit; feltlos, oder mit einzelnen Fettläppchen durchsetzt. In dem Czerny'schen Falle (Lit. Fall 9) fand sich ein Theil der Stränge in eine colossale elephantiastische Geschwulst eingelagert.

Der zweite wesentliche Bestandtheil der Geschwulst, die Stränge, zeigten meist einen ziemlich kreisförmigen Querschnitt, bisweilen erschienen sie abgeplattet. Sie waren meist wurmartig geschlängelt, stellenwejse um die Axe gedreht, und von sehr verschiedener Dicke. (In einigen Fällen schwankte dieselbe zwischen Bruchtheilen eines Millimeters und einigen Millimetern, in anderen ging sie bis zu $1 \mathrm{Cm}$, in noch anderen erreichten die Gebilde selbst einen Durchmesser von mehreren Centimetern.) An den einzelnen Strängen selbst wechselte der Durchmesser fortwährend, bald traten dicke, knotige Anschwellungen auf, bald verliefen die Stränge eine Strecke weit in nahezu cylindrischer Form. Zuweilen zeigten sie dicke kolbige Zweige, zuweilen setzten sie sich in feine Fäden fort. Sie waren, wenn sie im Unterhautzellgewebe oder in der Haut selbst lagen, stets durch zahlreiche Anastomosen verbunden und bildeten hier somit wahre, netzartige Plexus. Stets zeigten sie sich vielfach verzweigt. In einigen Fällen traten die Zweige in Haut oder Muskeln 
ein. Thre Farbe war röthlich - bis gelblichweiss, ihre Consistenz fest und derb, ihre Zerfaserung wegen der grossen Zähigkeit ihrer Substanz schwierig. Auf dem Durchschnitl erkannte man im Centrum oft einen dunkleren Punkt, umgeben von einer weisslichen, glänzenden, concentrisch angeordneten Masse. Diese peripherische Schicht liess sich bisweilen gleich einzelnen Häuten abziehen.

In einigen Fällen liess sich der Zusammenhang der Stränge mit wirklichen Nervensträngen deutlich nachweisen. Es liess sich hier erkennen, dass die Stränge nichts Anderes waren, als die verdickten Nerven selbst. Meist waren spinale; in einem Falle auch sympathische Nerven befallen. Die Anordnung der Stränge entsprach somit im Ganzen der der normalen Nervenverzweigungen; wo letztere Geflechte bilden, zeigten auch die Geschwulststränge eine eigentlich "plexiforme" Anordnung. Die stellenweise bedeutende Schlängelung und Torsion der Geschwulststränge deutet darauf hin, dass mit der Verdickung gleichzeitjg eine Verlängerung der Nerven stattgefunden hat.

Wo die Geschwulst unter oder in der Haut lag, war diese oft verdickt, zuweilen bräunlich bis rostfarben pigmentirt, manchmal auch mit hypertrophischen Haaren besetzt. In 2 Fallen bestand Eczem.

\section{Feinere Anatomie (Mikroskopisches).}

Bei genanerer Untersuchung ergab sich, dass das interstitielle Gewebe zwischen den Strängen aus faserigem Bindegewebe von lockerem Bau bestand. Die Züge desselben ordneten sich in der Umgebung der Stränge concentrisch um diese an und waren von letzteren selbst durcb den wohlerhaltenen Lymphraum, an dem selbst Endothel nachgewiesen werden konnte, getrennt. In Czerny's Falle hatte das Gewebe der Geschwulst zum Theil einen myxomatösen Charakter. Verneuil giebt an, dass er in seinem Tumor junges Bindegewebe gefunden habe. Oft fanden sich in der Zwischensubstanz wohlerhaltene Fetlzellenhäufchen. Gefässe waren darin nicht selten

Die Stränge, als der wesentlichste Theil der Geschwulst, zeigten meist eine peripherische hellere Zone, die aus concentrisch angeordnetem, fibrillärem Bindegewebe bestand. Nach innen von diesem folgten lockere Bindegewebszüge, die mehr verworren waren, 
im Centrum aber eine ziemlich longitudinale Richtung annahmen. Sie waren stets von zahlreichen Kernen begleitet. Besonders im Centrum, zuweilen aber unregelmässig vertheilt, fand man bei dünneren Strängen meist markhaltige Nervenfasern auf allen Stufen des Zerfalls; zum Theil noch wohlerbalten. Auch marklose werden mehrfach angegeben. Durch die Stränge verliefen mehr oder weniger zahlreiche Gefässe. In den stärksten Strängen waren die Nervenfasern sehr spärlich oder fehlten ganz.

Die Haut, welche die Geschwulst bedeckte, befand sich meist im Zustande einer merklichen Hypertrophie, die besonders das Bindegewebe der Cutis betraf. In einigen Fällen waren Talgdrüsen und Haarfollikel hypertrophisch. Die Epidermis zeigte öfters in den tieferen Schichten des Rete Malpighi eine Pigmentablagermng (um die Kerne der Zellen). Aus mehreren Beobachtungen geht hervor, dass die beschriebenen Stränge selbst bis in die Cutispapillen vordringen.

Ueber das feinere Detail der gesammten Veränderung, besonders bezüglich der Entwickelung der Geschwulst, gehen die Angaben bedeutend auscinander.

Czerny und Winiwarter nebmlich schildern eine Vermebrung der Zellen der Gefässwand bei Capillaren, Arterien und Venen, welche ebenso die im Unterhautzellgewebe und der Haut, wie die in den Nerven selbst verlaufenden Gefässe betraf und nach Winiwarter's Angabe besonders zur schliesslichen Neubildung von Bindegewebe führen soll, indem die von der Gefässwand gebildeten Spindelzellen endlich in Bindegewebsfasern übergehen sollen. Eine gleiche Wucherung fand sich nach Winiwarter an den Gefässen der Muskeln. An der Schwann'schen Scheide der Nervenfasern, wie auch am Sarcolemma der Muskelfasern wurde von ihm eine ähnliche Zellenproliferation beobachtet; auch diese soll zur Bildung von Bindegewebe führen. Das interstitielle Bindegewebe $z$ wischen den Nervenfasern in den Nervenstämmen soll dagegen nur eine geringe Vermehrung erfahren. Ebenso das Perimysium. In den Capillaren soll es infolge der Kernwacherung und Obturation des Lumens durch dieselbe zur Thrombose kommen, die eine Pigmentirung der umgebenden Gewebspartien zur Folge haben soll. So erklärt W. die von inm beobachtete Pigmentirung des Rete Malpighi einer Hautstelle, 
Czerny und Winiwarter stellen die Hypothese auf, dass die ganze Affection auf einer Erkrankung der trophischen Nerven der betroffenen Gewebe beruhe, wobei Czerny sich auf die Betheiligung des Sympathicus in seinem Falle stützt ${ }^{1}$ ).

Als ein böchst bemerkenswerther Befund würde, wenn weitere Beobachtungen ihn über jeden Zweifel erhöben, die schon von Bruns angegebene, von $\mathrm{Czerny}$ und Winiwarter bestätigte Neubildung von Nervenfasern aus Bindegewebszellen, resp. „Spindelzellen" von eigenthümlicher Beschaffenheit in der Geschwulst sein. Ob der Schluss berechtigt ist, dass hier in der That neugebildete Nervenfasern vorlagen, wage ich nicht $\mathrm{zu}$ entscheiden; jedenfalls jst es wünschenswerth, dass er durch weitere Beobachtungen bestätigl werde. In unseren Fällen fehlen alle Anbaltspunkte für eine solche Annabme, so dass sie jedenfalls nicht als für alle plexiformen Neurome gültig betrachtet werden könnte. Auch von mir wurden einzelne eigenthümliche Spindelzellen beobachtel; doch hatten diese nichts wesentlich von Bindegewebszellen Abweichendes. Namentlich fehlte es gänzlich an Uebergangsformen zu Nervenfasern, während andererseits die in der Geschwulst vorhandenen Nervenfasern im Verlauf der Entwickelung, wie oben gesagt, deutlich abnahmen und atrophirten.

Im Uebrigen gelang es bei den erstbeschriebenen beiden Fällen, eine Kernwucherung in den Gefässwandungen nur zum Theil, eine solche in der Schwann'schen Scheide der Nerven gar nicht nachzuweisen. Ueberhaupt muss der Modus der Bindegewebsentwickelung für diese beiden Fälle noch in suspenso bleiben. Jedenfalls geht aus dem oben Angegebenen hervor, dass unsere Geschwülste eine Bindegewebsentwickelung im Verlauf der Nerven darstellten, durch die allmählich die Nervenfasern selbst zu Grunde gerichtet wurden. Allem Anscheine nach ging in diesen beiden Fällen die Wucherung allein vom Perineurium aus, unter Betheiligung der Gefässwandungen, und zwar sowohl von der Gesammthülle der Nerven, als den die einzelnen Nervenfasern trennenden Partien derselben. Gleichzeitig kommt eine mehr oder weniger starke Wucherung des den Nerven benachbarten Bindegewebes zu Stande, die

1) Das Nähere über die Veränderungen an Gefăssen und Nerven, sowie ïber die angedeutete Hypothese $s_{\text {。 }}$ bei Cizerny und Winiwarter a. $a_{0} 0$. 
oft zu einem förmlich elephantiastischen Zustande desselben, in einem Falle zu wirklicher Elephantiasis führte.

Somit lässt sich die vorliegende Geschwulstform - wenn wir von den drei Fällen, in denen Neubildung von Nervenfasern angegeben wurde, vorläufig abstrahiren - einmal mit den Fibromen, andererseits mit der. Elephantiasis congenita in Zusammenhang bringen.

Hier würden sich auch Fälle anreihen lassen, in denen eine den plexiformen Neuromen äbnliche Geschwulstbildung an Nerven bei erworbener Elephantiasis oder ähnlichen erworbenen Geschwälsten vorkam. Dahin könnte auch der oben mitgetheilte fall von Verneuil (Lit. Fall 3) gerechnet werden, da hier der Zustand wohl sicher erworben war. Ein anderer hierher zu ziehender Fall wäre ein von $\mathrm{Chelius}^{1}$ ) veröffentlichter.

Hier betraf die Verdickung den N. tibialis, der sehr stark, zum Theil rosenkranzartig angeschwollen war, während gleichzeitig auf traumatischem Wege erworbene Elephantiasis cruris bestand.

In einem von $\mathrm{Barkow}{ }^{2}$ ) beschriebenen Falle war bei einem Manne auf den Stoss eines Ochsen gegen die Wade eine Geschwulst entstanden, die in einigen Jahren 3 Fuss Umfang erreichte. Bei der genaueren Untersuchung fanden sich in derselben Anschwellungen des $\mathrm{N}$. tibialis und peronens. Besonders der $\mathrm{N}$. tibialis war sehr stark verdickt. Gleichzeitig iand sich Neurombildung an den Verzweigungen der Nn. vagi.

Die Strangbildungen würden wir den "falschen Neuromen" Virchow's unter der Bezeichnung ,plexiformes Fibrom der Nervenscheiden" einreihen können, in den Fällen wenigstens, wo sicher keine Nervenneubildung stattfand, indem durch die Bezeichnung "plexiform" zugleich ausgedrückt werden soll, dass die Fibrombildung die Nerven über grössere Strecken bin contjnuirlich begleitet, wăhend die Bildung eigentlicher Plexus jedenfalls nur als ein zufälliger, nach der primären Anordnung der Nerven variirender Factor zu betrachten ist. Ebenso gut oder vielleicht besser könnte man für diese Fälle den von Verneuil angegebenen Namen: "Neuroma cylindricum plexiforme" mit verwerthen, indem man die

) S. Chelius, Heidelberger klin. Annalen. Bd. II. S. 354. Taf. I-II.

2) S. Verbandl, der Kais. Leopold. Karolin. Acad. der Naturf. Bd. VI. Abth. 2. Bonn 1829. S. $517-544$. 
Geschwulst als „cylindrisches Fibrom der Nervenscheiden“ bezeichw nete. Hier würde man freilich von den beträchtlichen Verschiedenheiten in der Dicke jedes einzelnen Stranges absehen. Etwas unbestimmter wäre der Ausdruck: „Strangförmiges Fibrom der Nervenscheiden".

Die Geschwulst würde nach der gegebenen Auffassung einen Beleg für die von Billroth ${ }^{1}$ ) schon vor Jahren aufgestellte Ansicht darbieten, dass gewisse Geschwülste, besonders aus der Sarcomreine (mit Einschluss der Fibroide), sich in ihrer Entwickelung genau an präformirte Formen anschliessen, seien dies bun Gefässe oder Nerven. Billroth führt mehrere derartige Fälle an, die den unsrigen ziemlich analog waren. Im Centrum der einzelnen Stränge oder Knoten fanden sich Gefässe oder Nerven. Zwei ähnliche Fälle von „plexiformen Geschwülsten" haben Billroth und

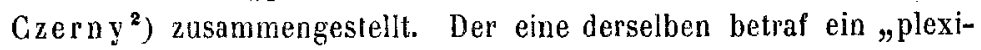
formes Myxosarcom " aus der Orbita, dessèn stricknadeldicke, vielfach verflochtene, anastomosirende und sich verästelnde Stränge im Centrum je ein Blutgefäss einschlossen; der andere ein „plexiformes, ossificirendes Chondrofibrom" des Oberkiefers, welches plexusartig zusammenhängende gewundene Stränge und Kuoten aus sehr festem, zum Theil knorpelhartem Gewebe zeigte, die selbst im Centrum verknöchert waren, von lockerem, gefässhaltigem Bindegewebe umgeben. Hier waren Gefässe in den Strängen nicht nachzuweisen,

Am Schlusse meiner Arbeit bleibt mir nur noch die angenehme pflicht, meinen hochverehrten Lehrern, den Herren Proff. Graefe und Volkmann für die freundliche Ueberlassung der Präparate, sowie Herrn Prof. Ackermann für die gütige Erlaubniss, in seinem Institut zu arbeiten, wie endlich Herrn Prof. Stẹdener für seine freundlichst und bereitwillig gewährte Unterstïtzung meinen herzlichsten Dank zu sagen.

1) S. Billrotb, Deber die Entstebung der Fibroide. Langenbeck's Archiv Bd. IV. 1863. S. 545 .

2) S. Billroth und Czerny, Ueber plexiforme Geschwälste. La agenbeck's Archiv Bd. XI. S. 230. 


\section{N a c h t r a g.}

Gleich nach Beendigung der obigen Arbeit erschien eine $A b-$ handlung von Dr. A. (arta ${ }^{1}$ ), in der sich ansser mehreren der oben genannten Fälle folgende, in Frankreich zur Beobachtung gekommene verzeichnet finden:

1. Robin, Bulletins de la Soc. de biol, 1854. Plexiformes Neurom des Plexus solaris bei einem Manne von 45 Jahren.

2. Guersant, Bull. de la Soc. de chir., 1859. Plexiformes Neurom in der Regio mastoidea bei einem Mädchen von 13 Jahren. Scheinbar ein ebensolches am Gaumengewölbe.

3. Christot, Gazette hebdom., 1870. Plexiformes Neurom der Nackengegend bei einem Mädchen von 18 Jahren.

4. Laroyenne, bei Christot angeführt. Plexiformes Neurom an der rechten Wange bei einem Knaben von 5 Jahren. Uebergang in Sarkom.

5. Verneuil, bei Gbristot. Plexiformes Neurom in der Gegend der Brustdrüse.

6. Cartaz, Étude sur la nérr. plexif. 1. c. Plexiformes Neurom binter dem linken $0 \mathrm{hr}$ in der Regio occipito-parietalis bei einem Knaben von 11 Jahren.

Diese 6 Beobachtungen würden die Zahl der bekannten Fälle auf 18 erhöhen. In Betreff des Sitzes der Gescbwulst ist ihr Vorkommen am Plexus solaris und an der Brustdrüse bemerkenswerth. Der sub 1 angegebene Fall ist noch dadurch besonders merkwürdig, dass die Geschwulst bei einem Manne von 45 Jahren zufällig bei der Section vorgefunden wurde und während des Lebens durchaus keine Störungen veranlasst hatte.

In einem Falle wurde vollständige Anäsihesie der Geschwulst beobachtet, so dass selbst Nadeln bis auf's Blut eingestochen werden konnten, obne dass der Patient es fühlte.

Ein Recidiv wurde in dem sub 4 verzeichneten Falle beobachtet. Hier war die Geschwulst sarcomatös degenerirt. Die Möglichkeit einer solchen Degeneration würde, wenn öfters beobachtet, die Prognose der Geschwulst natürlich wesentlich verschlechtern.

Bezüglich der Diagnose hebt Cartaz besonders hervor, dass eine Verwechselung mit Lipom möglich sei, ein Irrthum, der in der That mehrfach vorgekommen ist. In anatomischer Hinsicht betont Cartaz die gleichzeitige Vermehrung der Nervenfasern und des Bindegewebes der befallenen Nervenstränge, giebt freilieh zu, dass

1) Dr. A. Cartaz, Étude sur le névrome plexiforme. Archives générales de Médecine, מuméro d'aout 1876. 
durch secundäre Atrophie der ersteren die Geschwulst zum reinen Fibrom werden könne. Im Ganzen charakterisirt er sie als Fibroneurom und behält die Verneuil'sche Bezeichnung: „Neuroma cylindricum plexiforme" bei. Dass in den genannten Fällen wirklich eine Vermehrung der Nervenfasern stattgefunden hat, scheint indessen nach den vorliegenden Beobachtungen nicht vollständig sicher zu sein.

\section{Erklärung der Abbildungen. \\ Tafel $I$.}

Fig. 1. Ein Stück der Geschwulst von Fall 1, aus dem oberen Augenlid und der Augenböhle. (Natürliche Grösse.) Das die Stränge umbüllende Bindegewebe ist mit Ausnabme einer Partie (b) entfernt und es sind die im Ganzen cylindrischen, mehrfach anastomosirenden Stränge sichtbar. Ein feiner Nerv (a), der in die Geschwulst eintritt, schwillt (bei c) plötzlich beträchtlich an.

Fig. 2. Querschnitt durch einen stärkeren Strang aus einer anderen Partie derselben Geschwulst. (Hartnack. 0bj. J. 0c. 2.) Carminpräparat. Der Querschnitt des grossen Stranges $\left(a_{1}\right)$ zeigt besonders in der Peripherie eine deutlich concentrische Schichtung. Bei $a_{2} a_{2}$ sind 2 kleinere Strănge im Querschnitt getroffen. In diesen findet sich noch je ein Bündel von Nervenfasern, die im grossen Strange $a_{1}$ nicht mehr sichtbar sind. b Lymphraum. c Arterien im Querschnitt, d Fett.

Fig. 3. a Ein Theil der Mitte eines ziemlich dicken Stranges im Querschnitt bei stärkerer Vergrösserung. (Hartnack 0bj. VHI. Oc. 3.) Picrocarminpräparat. Man erkent einzelne wellige Züge von Bindegewebe, dazwischen Bindegewebsmassen, die sich in mannichfachen Windungen zwischen einzelnen wohlerhaltenen Nervenfasern $(a)$ bindurchschlingen. Zahlreiche dunkelgefärbte Kerne sind im Bindegewebe sichtbar, stellenweise im Querschnitt getroffen. b Querschaitt durch einen dünnen Nerven, der die Bindegewebsentwickelung im Beginn zeigt. (Hartnack 0bj. IV. 0c. 3.) In der Peripherie findet sich eine concentrisch geschichtete Bindegewebshülle. Die einzelnen Nervenbündel sind in der rechten Hälfte des Querschnittes noch wohl erhalten; in der linkes dagegen sind zwar die Umrisse der Bündel noch wohl erbalten, die Nervenfasern in denselben jedoch zu Grunde gegangen und durch Bindegewebe ersetzt. Zwischen den Bündeln sind die Lymphräume deutlich.

Fig, 4. Einzelne Bestandtheile von Zerfaserungspräparaten, nach Hartnack 0bj. X à Immers., $0 c .3$ gezeichnet. a $a_{1} a_{2} a_{3}$ Spindel- und sternförmige Zellen aus dem Bindegewebe elnes mässig dicken Stranges. b Spindelzelle mit langen Ausläufern. c Feine Nervenfaser mit spärlicher, granulirter Markscheide. Der Axencylinder ist deutlich. d Nervenfaser. Axencylinder $(\gamma)$ erhalten. $\mathrm{Um}$ denselben (bei $\beta \beta$ ) das granulirte Mark stellenweise in grösserer Menge angelaäuft. Bei $\alpha$ ein Kern im Neurilemma, e Bindegewebszug mit einzelnen Spindelzellen. 